\title{
Design and implementation of the noise sensor signal conditioning
} Guangdong $\operatorname{Han}^{1, a *}$, Hang Li ${ }^{1, b}$, Haiquan Chen ${ }^{1, c}$, Yuqing Sun ${ }^{1, d}$, Jinnan Zhang ${ }^{1, e}$, Shanghai wang ${ }^{1, f}$, Zhuanzhao $\mathrm{Liu}^{2,9}$

${ }^{1}$ Marine Engineering College, Dalian Maritime University, Dalian China

${ }^{2}$ ShanDong Jiao Tong University

a18842602951@163.com; 9Izz9000@aliyun.com

Key words: noise sensor; LM285 integrated audio to put the AD620 differential amplifier; reliability

Abstract: the design of signal conditioning circuit module is mainly used for weak signal sensor noise amplification and regulation, the module uses LM386 integrated audio amplifier for signal preamplifier, using precision rectifier converts the amplified signal to the DC output, at the same time using differential amplifier with differential amplifier AD620, AD620 is actually a difference the amplifier offset voltage, the current is small, high common mode rejection ratio, so the processing of weak signal, with excellent performance that is amplified and eliminate the noise. To improve the measuring accuracy and reliability of sensor noise.

\section{Introduction:}

Signal conditioning circuit modules are widely used in industrial control, instruments and meters, et of the sensor signal detection and processing applications. It is a sensor and A/D converter device such as a follow-up of the interface. It will be very weak of sensor output and low level signal is amplified by a variety of noise interference, processing, noise elimination and unified format, make it suitable for A/D converter device such as a subsequent request signal. For different object to be tested and used by many different kinds of sensors, the signal conditioning circuit is put forward different requirements. The follow-up device such as A/D converter combining with technical indicators to design a good performance of signal conditioning circuit has become the key content of system design.

Noise interference has a strong sex, I am in a device measuring the noise decibels are vulnerable to the surrounding noise sound, this would require the receiver noise has voice recognition function, but it is not so, according to the design is too complicated, it is the problem with a low resolution microphone microphones can solve, to do so is not only economic use.

Special funds for basic scientific research business of the Central University (132014332,3132015025); Liaoning Natural Science Foundation of China(2015020132) 


\section{Basic operation principle}

\section{The principle diagram of the device}

\section{Microphone}

microphones

\section{The prefix \\ amplification}

Precision

rectifier
The suffix

amplification

Fig.1 The principle diagram of the device as a whole

The principle diagram of the device as a whole show in fig.1.From it we can know the whole idea of this paper.

\section{Introduction of the principle}

The noise in front of the microphone microphones will environment into a faint millivolt level analog voltage signals, the voltage signal through the front part stage amplifier amplification, enlarged by precision rectifier circuit, the ac voltage signal is converted into dc signal, the signal again after the amplifier section, zoom into 0 to $5 \mathrm{v}$ analog voltage signal, and then the signal is sent to the digital display control instrument. Regulate the range of parts and the zero point can be adjusted through the potentiometer.

\section{Unit Analysis}

\section{Signal conditioning part}

This part by stage amplifier, precision rectifier part before and after the amplifier components. Detector receiver microphones will be in the environment noise into a faint millivolt level analog voltage signals, the voltage signal through the signal conditioning part into 0 to $5 \mathrm{v}$ analog voltage signal, and the analog alarm display adjustment to the section.

\section{Before the amplifier application}

Stage amplifier part before the electret microphone acquisition of speech signal, convert its signal amplitude is millivolt level of electrical signals, system level before the treatment, as far as possible improve signal to noise ratio at the input to the amplifier, guarantee the gain amplifier circuit with precise, stable. Figure.2 Former stage amplifier circuit diagram. Using operational amplifier LM386, 2 for inverting input pin, 3 for the same phase input pin, pin 5 for output, pin 6 and 4 respectively the power and ground, pin 1 and 8 for voltage gain setting, when used in pin 7 and indirect bypass capacitors in the land. In indirect capacitance of pins 1 and 8 (pin 1 pick capacitance is level) to change the gain.

Capacitor C3 is pin 7 bypass capacitors, it can have the effect of filter out noise;

Resistance of R3 and resistor R1, bleeder circuit provide relatively stable power supply to the MIC;

Capacitor C5 is the output coupling capacitor, the capacitor is used to partition the dc voltage and coupling of audio communication signals, it constitutes the first-order high-pass filter and load. 


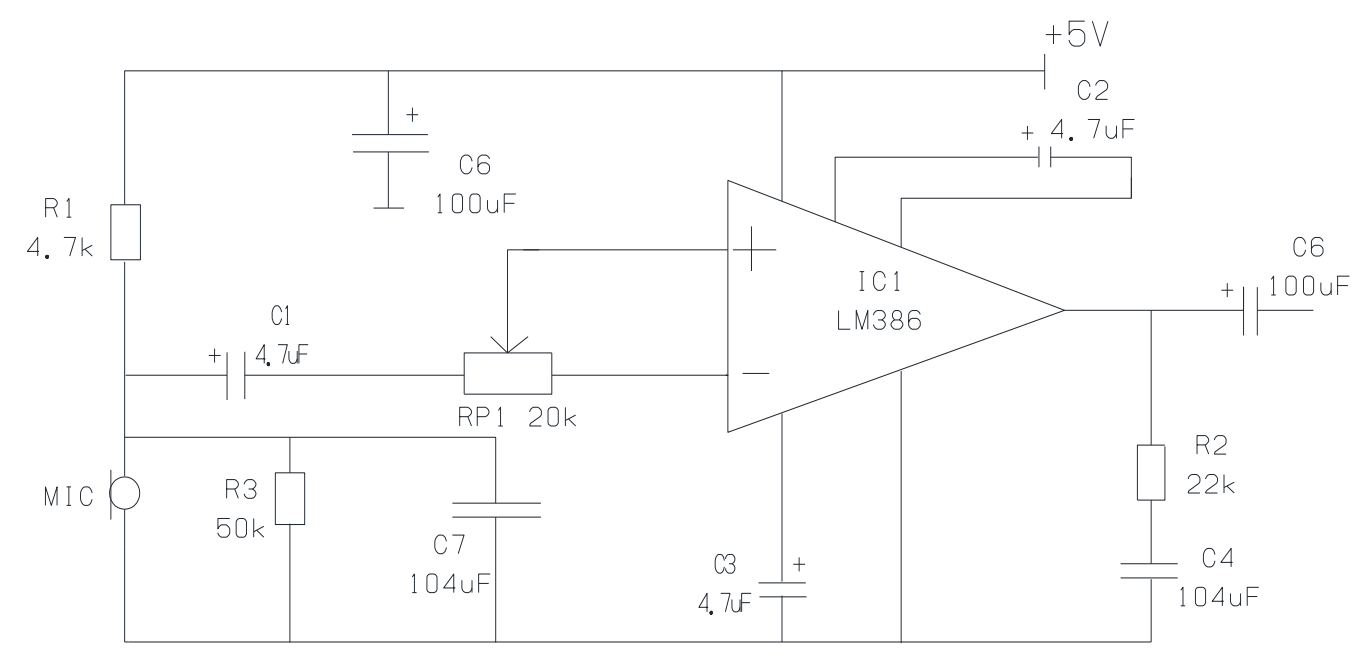

Figure.2 Former stage amplifier circuit diagram

\section{Precision rectifier part}

To convert ac signal into de signal must adopt rectifier, precision rectifier circuit diagram show in figure3. The conventional method is to use diode rectifier directly, but due to the diode rectifier threshold voltage $(0.3 \mathrm{~V}$ or $0.3 \mathrm{~V})$, small signal rectifying error is big, can't even work. To weak alternating current (ac) is converted into unidirectional pulse of electricity, it is important to adopt precision rectifier, so the circuit must be composed by op-amp and diode to achieve precision rectifier.

We used in this part of the operational amplifier LM358, its internal consists of two independent, high gain, internally frequency compensated dual operational amplifier, two channels to deal with the positive and negative half cycle, regardless of the input is a positive or negative, the output is positive voltage.

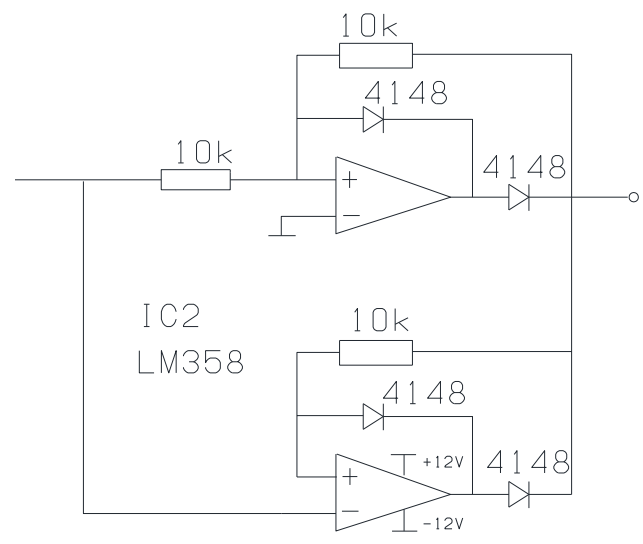

Figure. 3 precision rectifier circuit diagram

\section{After the amplifier application}

After stage amplifier circuit diagram show in figure4. After stage amplifier adopts differential amplifier to achieve the required drive ability, its advantage lies in the differential amplifier can get higher common mode rejection ratio, strengthen the anti-interference circuit.

Differential amplifier differential amplifier AD620 was adopted to realize. Internal is actually a difference amplifier AD620, the small disturbance voltage current, common mode rejection ratio is high, so deal with weak signal, which is amplified and eliminate noise, excellent performance, it can gain by adjusting the resistance between pins 1 and 8 , its gain $\mathrm{G}=1+(49.4 \mathrm{k} \Omega / \mathrm{Rg})(\mathrm{Rg}$ in the resistance between pins 1,8 ). 
AD620 of 6 pins at the back of the part is the conventional RC low-pass filter. R304 and R305 can be considered a current limiting function, can also be considered to be the separation.

OP07 precision op-amp is zero reference source, mainly serving the AD620, provide a reference for AD620 voltage, OP073 pin number at the back of the part for precision voltage regulator, to OP07 provide a stable source of unknown to zero.

Through the above three parts, we can send the electret microphone acquisition of speech signal is converted into 0 to $5 \mathrm{v}$ analog voltage signal, and then send it to display debug apparatus.

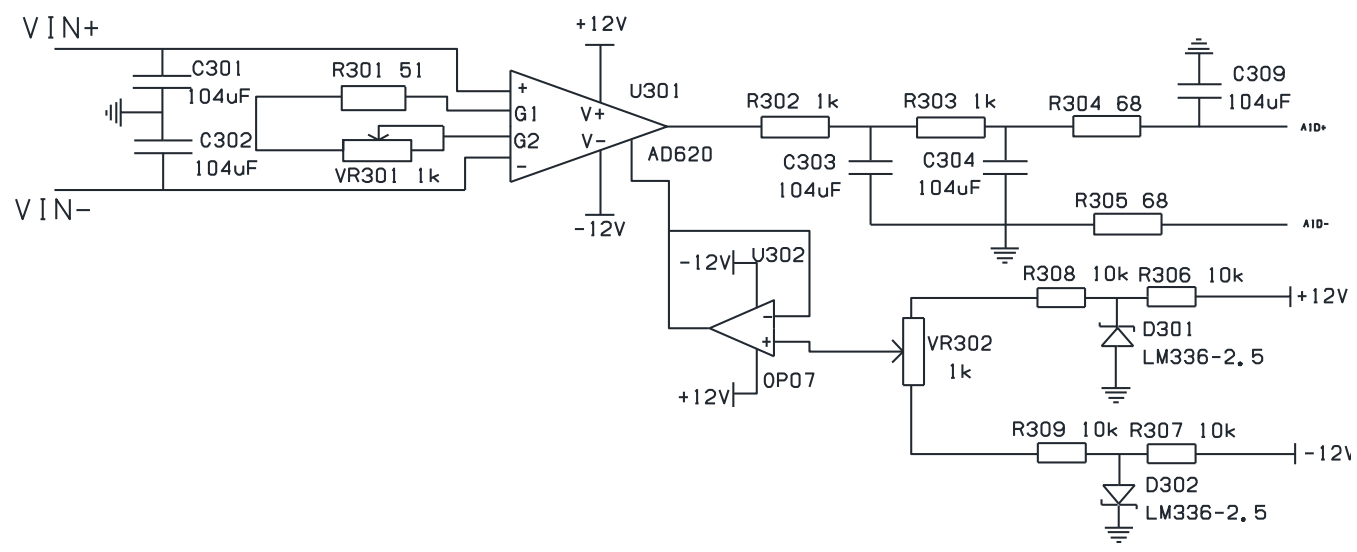

Figure.4 After stage amplifier circuit diagram

\section{Physical figure and the experimental data}

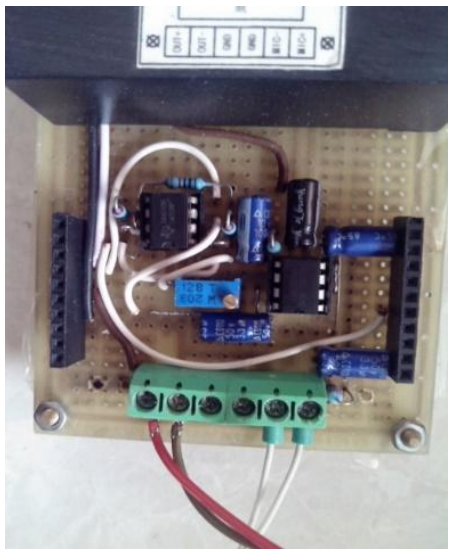

Figure.5 Former stage amplifier and precision rectifier

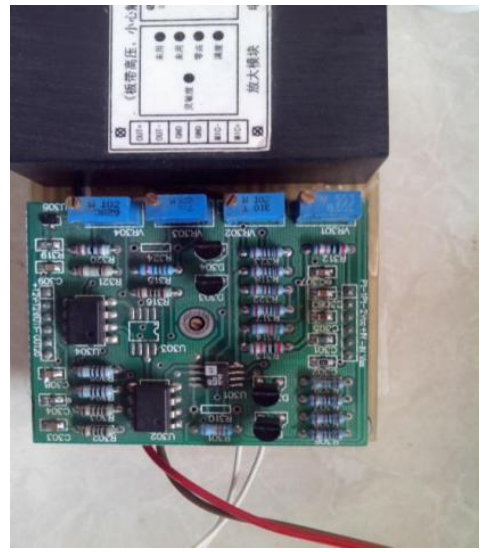

Figure.6 After stage amplifier

Figure 5 and figure6 show the object of former stage amplifier and precision rectifier and after stage amplifier

In the experiments, the collected Marine auxiliaries an audio noise, the parameters for quantitative figures for $6000 \mathrm{~Hz}, 16$ (resolution), or long for $5 \mathrm{~s}$ (about thirty thousand sampling points), this is the time domain waveform. The noise figure show in the figure 7.Obviously cannot see the signal from the characteristics or parameters.

In view of the signal processing is shown in figure 7 . The processed signal is show in figure 8. One black for the original signal, a red signal after conditioning, obviously, it can be seen from the 
figure that the cycle of the original signal characteristics are not obvious, but after the signal has the obvious cyclical and smoothness.

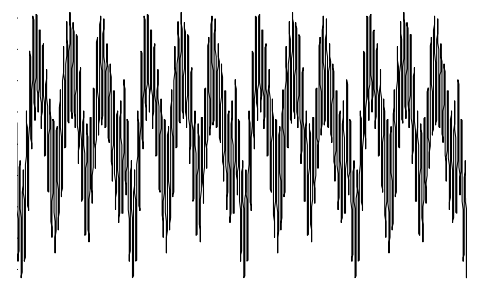

Figure.7 Time domain noise figure

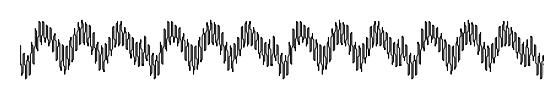

Figure.8 After the signal processing of waveform figure

\section{Conclusions}

Noise analysis is presented in this paper, on the basis of the design of the signal conditioning circuit are discussed in detail, points out the structure and the parts of the circuit components selection, experimental results of the microphone signal detection is presented. Experiments show that: the signal conditioning circuit with stable performance, high reliability, strong flexibility and the advantages of programmable, fundamentally solves the previous signal disposal device performance is not stable, affected by the environment, and the problem of small dynamic range. This circuit technical indicators are as follows: (1) the dynamic range: a few microvolts to dozens of millivolt; (2) sensitivity: $1 \mathrm{lv}$; (3) response time: better than $1 \mathrm{~ms}$.

\section{Acknowledgements}

Special funds for basic scientific research business of the Central University (132014332,3132015025); Liaoning Natural Science Foundation of China(2015020132)

\section{References}

[1]ZENG Qing-yong. Weak Signal Detection [M]. Hangzhou: Zhejiang University Press, 1996. $1 \sim 83$.

[2] CHEN Jiang-hua. Analysis and Study on the Noises in Digital VISI Circuits [J]. Journal of Shandong University of Technology, 2000, 30 (4): 367 372. 
[3] LIU Jian, QINHu-i bin. MAX260/261/262 Programmable Switched-Capacitor Filters and Its Application [J]. Journal of Hangzhou Institute of Electronic Engineering, 2003, 23 (6): 19 22.

[4] ZHANG Jin-1, i JING Zhan-rong, LIANG, et all Weak Signal Conditioning Circuit Design and Noise Estimation [J]. Electronic Measurement Technology, 2007, 30 (11): 40 42.

[5] WU Gang, ZHANG Xiao-ping. Research of Resistance Thermal Noise [A]. Ordnance Industry Automation, 2006, 25 (4): 86 87.

[6] HOU Jian, LI Yong-hong. Design of Signal Processing Circuit in ECG Monitoring System [J]. Industrial Control Computer, 2009, 22 (1): 81 84. (In Chinese) 